VOL. $71(2005)$ [459-470]

\title{
ON THE COHOMOLOGY OF EXTENSIONS BY A HEISENBERG LIE ALGEBRA
}

\author{
Hannes Pouseele
}

\begin{abstract}
This article describes the cohomology spaces of any Lie algebra containing a Lie algebra of Heisenberg type (whose cohomology was studied by Santharoubane) as an ideal of codimension 1. For instance, the twisted standard filiform Lie algebras are of this kind. We give an explicit formula for the Betti numbers of this Lie algebra, and use this to describe new families of algebras whose Betti numbers do not behave unimodally.
\end{abstract}

\section{INTRODUCTION}

Although defined in linear algebra terms, the cohomology of a Lie algebra remains difficult to describe. Even in determining the Betti numbers $\beta_{i}=H^{i}\left(\mathfrak{g}_{n}, \mathbb{R}\right)$ of the Lie algebras in a family $\left\{\mathfrak{g}_{n}\right\}_{n}$, one meets serious combinatorial problems (see for instance $[2,3,7])$. To handle these, we use spectral sequence type arguments, thus relating the cohomology of the Lie algebra we are interested in, to a Lie algebra whose cohomology has already been studied.

Let $h_{n}$ be a (real) Heisenberg Lie algebra with a basis $\left\{x_{1}, \ldots, x_{n}, y_{1}, \ldots y_{n}, w\right\}$ and corresponding Lie bracket

$$
\left[x_{i}, y_{i}\right]=w
$$

for all $1 \leqslant i \leqslant n$. The following theorem is the central result of this article.

THEOREM. (With notations as above.) Let $\mathfrak{g}$ be an extension of the one-dimensional Lie algebra $\langle z\rangle$ by $\mathfrak{h}_{n}$, for some $n$,

$$
1 \longrightarrow \mathfrak{h}_{n} \longrightarrow \mathfrak{g} \longrightarrow\langle
$$

Received 17th December, 2004

I would like to thank Prof. Dr. Dietrich Burde (Universität Wien) for his helpful suggestions. The figures in this article were made with Mathematica ${ }^{\odot}$.

Copyright Clearance Centre, Inc. Serial-fee code: 0004-9727/05 \$A2.00+0.00. 
such that $\mathfrak{g}$ acts trivially on the centre $\mathfrak{z}=\langle w\rangle$ of $\mathfrak{h}_{n}$. Let $\mathfrak{f}=\mathfrak{g} / \mathfrak{z}$. Then

$$
\beta_{p}(\mathfrak{g})= \begin{cases}\beta_{p}(\mathfrak{f}) & \text { for } p=0 \text { or } p=1 \\ \beta_{p}(\mathfrak{f})-\beta_{p-2}(\mathfrak{f}) & \text { for } 2 \leqslant p \leqslant n \\ 2\left[\beta_{n+1}(\mathfrak{f})-\beta_{n-1}(\mathfrak{f})\right] & \text { for } p=n+1 \\ \beta_{p-1}(\mathfrak{f})-\beta_{p+1}(\mathfrak{f}) & \text { for } n+2 \leqslant p \leqslant 2 n \\ \beta_{p-1}(\mathfrak{f}) & \text { for } p=2 n+1 \text { or } p=2 n+2\end{cases}
$$

The condition that $z$ acts trivially on the centre of $\mathfrak{h}_{n}$ is equivalent to the condition that $\mathfrak{g}$ is unimodular. In case $\mathfrak{g}$ is nilpotent, the action of $\mathfrak{g}$ on $w$ is automatically trivial. In general, this need not be the case; an easy counterexample is the extension of $\mathfrak{h}_{1}$ by $\langle z\rangle$ determined by

$$
\left[z, x_{1}\right]=x_{1}, \quad \text { and } \quad[z, w]=w .
$$

Picking an extension of $\langle z\rangle$ by $\mathfrak{h}_{\boldsymbol{n}}$ corresponds to choosing a derivation of $\mathfrak{h}_{\boldsymbol{n}}$; any derivation of $\mathfrak{h}_{n}$ has a matrix representation with respect to the basis given in (1) of the form

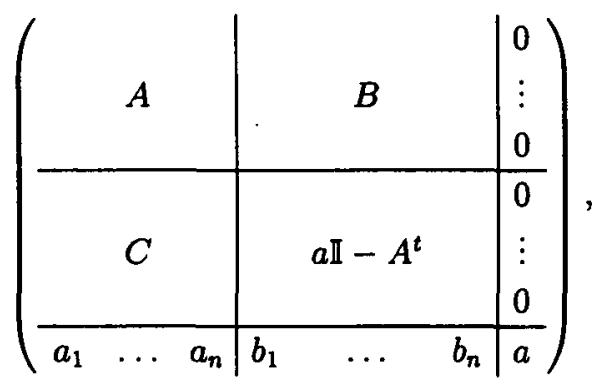

where $A, B, C \in \mathbb{R}^{n \times n}$ with $B$ and $C$ symmetric; $\mathbb{I}$ is the identity matrix of dimension $n$. In the setting of the main theorem, the parameter $a$ equals zero.

We apply our theory to two families of Lie algebras. First of all, we have a look at the algebras $\mathfrak{b}_{n}$ with a basis $\left\{x_{1}, \ldots, x_{n}, y_{1}, \ldots y_{n}, w, z\right\}$ and Lie bracket

$$
\left[z, x_{i}\right]=y_{i}, \quad\left[x_{i}, y_{i}\right]=w
$$

for all $1 \leqslant i \leqslant n$. We compute its Betti numbers in terms of the Betti numbers of the algebra $a_{n}$ determined by

$$
\left[z, x_{i}\right]=y_{i}
$$

with respect to a basis $\left\{x_{1}, \ldots, x_{n}, y_{1}, \ldots, y_{n}, z\right\}$. The Betti numbers of the algebras $\mathfrak{a}_{n}$ have been studied in [2]. An elementary analysis shows that, for any $n \geqslant 5$, the Betti numbers of $\mathfrak{b}_{n}$ do not behave unimodally (that is, $\beta_{i}\left(\mathfrak{b}_{n}\right) \leqslant \beta_{i+1}\left(\mathfrak{b}_{n}\right)$ for all $0 \leqslant i \leqslant n$ ), in contrast to, for instance, Lie algebras containing an Abelian ideal of codimension 1 (see [1]). 
The second type of Lie algebras we study, is the family of the twisted standard filiform Lie algebras $g_{n}$ given by

$$
\begin{array}{ll}
{\left[z, x_{i}\right]=x_{i+1},} & \text { for all } 1 \leqslant i \leqslant 2 n-1 \\
{\left[z, x_{2 n}\right]=w,} & \text { for all } 1 \leqslant i \leqslant n
\end{array}
$$

with respect to a basis $\left\{x_{1}, \ldots x_{2 n}, w, z\right\}$. Again, examples indicate that the Betti numbers of these algebras do not follow a unimodal distribution. The heart of the proof of this fact would involve studying a special type of partitions of integers, which is in itself an interesting problem, but is beyond the scope of this article.

\section{Cohomology of EXTENSIONS By the ONE-DIMENSIONAL LIE Algebra}

Let $\mathfrak{g}$ be any finite dimensional Lie algebra with a basis $\left\{x_{1}, \ldots, x_{n}\right\}$, and suppose $x_{n}$ is central. Let $\mathrm{pr}: \mathfrak{g} \rightarrow \mathfrak{f}=\mathfrak{g} /\left\langle x_{n}\right\rangle$ be the projection (also denoted by ${ }^{-}$). Then pr induces a map $\operatorname{pr}_{*}:\left(\Lambda^{*} \mathfrak{g}, d_{\mathfrak{g}}\right) \rightarrow\left(\Lambda^{*} \mathfrak{f}, d_{\mathfrak{f}}\right)$ of differential graded algebras, with $\Lambda^{*-1}\left\langle x_{1}, \ldots, x_{n-1}\right\rangle$ $\wedge x_{n} \cong \Lambda^{*-1} \mathfrak{f}$ as kernel. Applying the Hom(-, $\left.\mathbb{R}\right)$-functor to the short exact sequence of differential graded algebras

$$
0 \longrightarrow \Lambda^{*-1} \mathfrak{f} \longrightarrow \Lambda^{*} \mathfrak{g} \longrightarrow \Lambda^{*} \mathfrak{f} \longrightarrow 0
$$

and taking cohomology, induces a long exact sequence,

$$
\begin{aligned}
\ldots \longrightarrow & H^{p-2}(\mathfrak{f}) \stackrel{\mathrm{d}^{p-2}}{\longrightarrow} H^{p}(\mathfrak{f}) \stackrel{i^{p}}{\longrightarrow} H^{p}(\mathfrak{g}) \\
& \stackrel{j^{p}}{\longrightarrow} H^{p-1}(\mathfrak{f}) \stackrel{\mathrm{d}^{p-1}}{\longrightarrow} H^{p+1}(\mathfrak{f}) \longrightarrow \cdots
\end{aligned}
$$

Lemma 1. (With notations as above.) Let $u \in \Lambda^{2} \mathfrak{f}$ such that $i^{2}(u)=d_{\mathrm{g}}^{1} x_{n}^{*}$. Then the connecting homomorphism $\mathrm{d}^{p}: H^{p}(\mathfrak{f}) \rightarrow H^{p+2}(\mathfrak{f})$ is given by

$$
\mathrm{d}^{p}([f])=[f \wedge u] .
$$

PROOF: First of all, note that $j^{2}\left(d_{\mathfrak{g}}^{1} x_{n}^{*}\right)\left(\bar{x}_{i}\right)=d_{\mathfrak{g}}^{1} x_{n}^{*}\left(x_{i} \wedge x_{n}\right)=0$, such that $d^{1} x_{n}^{*}$ is contained in the image of $i^{2}$, and $u$ does exist. The connecting homomorphism $\mathrm{d}^{p}$ is induced on cohomology by following the dotted arrows in the diagram

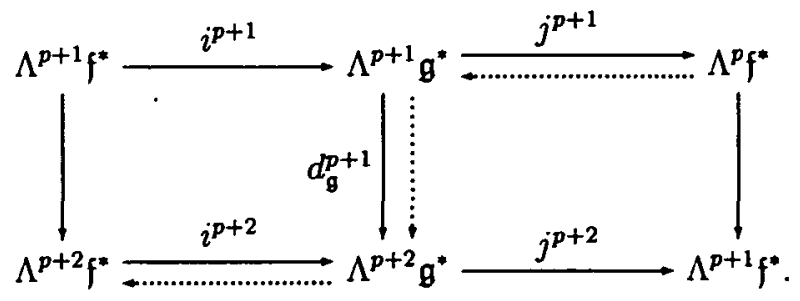


Let $f \in \Lambda^{p} \mathfrak{f}^{*}$ such that $d_{\mathfrak{f}}^{p} f=0$. Then $f$ lifts to an element $\bar{f} \in \Lambda^{p+1} \mathfrak{g}^{*}$ by defining

$$
\bar{f}\left(x_{i_{1}} \wedge \ldots \wedge x_{i_{p+1}}\right)= \begin{cases}f\left(x_{i_{1}} \wedge \ldots \wedge x_{i_{p}}\right) & \text { if } i_{p+1}=n \\ 0 & \text { otherwise }\end{cases}
$$

for any $1 \leqslant i_{1}<\cdots<i_{p+1} \leqslant n$. In other words, $\bar{f}=i^{p}(f) \wedge x_{n}^{*}$. Therefore,

$$
d_{\mathrm{g}}^{p+1} \bar{f}=i^{p}(f) \wedge d_{\mathrm{g}}^{1} x_{n}^{*}=i^{p+2}(f \wedge u) .
$$

EXAMPLE. Let $g_{1}$ be the twisted standard filiform Lie algebra of dimension 4 (see (2) for the description of a basis and its corresponding Lie brackets). We compute the cohomology of $\mathfrak{g}_{1}$ in terms of the cohomology of $\mathfrak{f}_{2}=\mathfrak{g}_{1} /\langle w\rangle$, the standard filiform Lie algebra of dimension 3 given by

$$
\left[z, x_{1}\right]=x_{2} \text {. }
$$

To do this, we need to compute the connecting homomorphisms $\mathrm{d}^{0}$ and $\mathrm{d}^{1}$. Since, for any $c \in H^{0}\left(\mathfrak{f}_{2}\right)=\mathbb{R}$, we have $\mathrm{d}^{0} c=c\left(z^{*} \wedge x_{2}^{*}-x_{1}^{*} \wedge x_{2}^{*}\right)$, $\mathrm{d}^{0}$ is injective. In degree 1 , a class $\left[c_{1} z^{*}+c_{2} x_{1}^{*}\right] \in H^{1}\left(\mathrm{f}_{2}\right)$ maps to $\mathrm{d}^{1}\left(\left[c_{1} z^{*}+c_{2} x_{1}^{*}\right]\right)=\left[-\left(c_{2}+c_{1}\right) z^{*} \wedge x_{1}^{*} \wedge x_{2}^{*}\right]$, so $\mathrm{d}^{1}$ is surjective. Now we know the connecting homomorphisms in the long exact sequence (3) relating the cohomology of $g_{1}$ to the cohomology of $f_{2}$, we know that

$$
\beta_{0}\left(g_{1}\right)=1, \quad \beta_{1}\left(g_{1}\right)=2, \quad \beta_{2}\left(g_{1}\right)=2, \quad \beta_{3}\left(g_{1}\right)=2, \quad \beta_{4}\left(g_{1}\right)=1 .
$$

It turns out this injectivity or surjectivity of the connecting homomorphisms in the long exact sequence (3) is typical for an extension of the one-dimensional Lie algebra by a Heisenberg Lie algebra $\mathfrak{h}_{n}$.

\section{Cohomology of extensions by a Heisenberg Lie algebra}

Let $\mathfrak{g}$ be an extension of $\langle z\rangle$ by the Heisenberg Lie algebra $\mathfrak{h}_{n}$,

$$
0 \longrightarrow \mathfrak{h}_{n} \longrightarrow 0
$$

such that the adjoined action of $z$ on the centre $\langle w\rangle$ of $\mathfrak{h}_{n}$ is trivial (see (1) for the description of the basis we use and its related bracket structure). Then $w$ is central in g. Write $\mathfrak{f}=\mathfrak{g} /\langle w\rangle$ for the quotient algebra. The long exact sequence (3) relates the cohomology of $\mathfrak{g}$ to the cohomology of $f$. In this long exact sequence, the connecting homomorphism $\mathrm{d}^{p}$ is given by the wedge product with

$$
u=z^{*} \wedge z^{\prime *}+\sum_{i=1}^{n} x_{i}^{*} \wedge y_{i}^{*}
$$

for some $z^{\prime} \in \mathfrak{f}$. Since there is no confusion here about the fact that we are working in the quotient algebra $\mathfrak{f}$, we do not mention the projections explicitly. 
Proposition 2. (With notations as above.) For each $p$, the connecting homomorphism $\mathrm{d}^{p}$ has maximal rank, that is, $\mathrm{d}^{p}$ is injective for all $p \leqslant n-1$, and $\mathrm{d}^{p}$ is surjective for all $n \leqslant p$.

We first of all use this proposition to prove the main theorem of this paper, postponing the proof of the proposition itself to the following section.

Proof of the MaIN TheOREM: First of all, since the five-term sequence

$$
H^{p-2}(\mathfrak{f}) \stackrel{\mathrm{d}^{p-2}}{\longrightarrow} H^{p}(\mathfrak{f}) \stackrel{i^{p}}{\longrightarrow} H^{p}(\mathfrak{g}) \stackrel{j^{p}}{\longrightarrow} H^{p-1}(\mathfrak{f}) \stackrel{\mathrm{d}^{p-1}}{\longrightarrow} H^{p+1}(\mathfrak{f})
$$

coming from (3), is exact, we know that

$$
\operatorname{dim} H^{p}(\mathfrak{g})=\operatorname{dim} \operatorname{Im} i^{p}+\operatorname{dim} \operatorname{Im} j^{p}=\beta_{p}(\mathfrak{f})-\operatorname{dim} \operatorname{Im} \mathrm{d}^{p-2}+\operatorname{dim} \operatorname{ker} \mathrm{d}^{p-1} .
$$

Now let $p \leqslant n$. Then both $\mathrm{d}^{p-2}$ and $\mathrm{d}^{p-1}$ are injective, so

$$
\operatorname{dim} H^{p}(\mathfrak{g})=\beta_{p}(\mathfrak{f})-\beta_{p-2}(\mathfrak{f}) .
$$

For $p \geqslant n+2$, both $\mathrm{d}^{p-2}$ and $\mathrm{d}^{p-1}$ are surjective, so

$$
\operatorname{dim} H^{p}(\mathfrak{g})=\beta_{p-1}(\mathfrak{f})-\beta_{p+1}(\mathfrak{f}) .
$$

In case $p=n+1$ we know that $d^{n-1}$ is injective and $d^{n}$ surjective, so

$$
\begin{aligned}
\operatorname{dim} H^{n+1}(\mathfrak{g}) & =\beta_{n+1}(\mathfrak{f})-\beta_{n-1}(\mathfrak{f})+\beta_{n}(\mathfrak{f})-\beta_{n+2}(\mathfrak{f}) \\
& =2\left[\beta_{n+1}\left(\mathfrak{f}_{n-1}\right)-\beta_{n-1}\left(\mathfrak{f}_{n-1}\right)\right]
\end{aligned}
$$

by Poincaré duality.

\section{A PROOF OF PROPOSITION 2}

In this section, we show that the map $\mathrm{d}^{p}=\mathrm{d}_{\mathfrak{f}}^{p}: H^{p}(\mathfrak{f}) \rightarrow H^{p+2}(\mathfrak{f})$ on the cohomology of $f$, induced by the wedge product with

$$
u=z^{*} \wedge z^{\prime *}+\sum_{i=1}^{n} x_{i}^{*} \wedge y_{i}^{*}
$$

has maximal rank.

Let $\mathfrak{a}$ be the Abelian ideal of $f$ generated by $x_{1}, \ldots, x_{n}, y_{1}, \ldots, y_{n}$. Then, according to [4], the cohomology of $f$ fits into a long exact sequence

$$
\begin{aligned}
\ldots \longrightarrow & H^{p-1}(\mathfrak{a}) \stackrel{\delta^{p-1}}{\longrightarrow} H^{p-1}(\mathfrak{a}) \stackrel{i^{p}}{\longrightarrow} H^{p}(\mathfrak{f}) \\
& \stackrel{j^{p}}{\longrightarrow} H^{p}(\mathfrak{a}) \stackrel{\delta^{p}}{\longrightarrow} H^{p}(\mathfrak{a}) \longrightarrow \ldots,
\end{aligned}
$$

where the connecting homomorphism $\delta^{p}: H^{p}(\mathfrak{a}) \rightarrow H^{p}(\mathfrak{a})$ is induced by the adjoined action of $z$ on $\mathfrak{a}$. 
LEMma 3. (With notations as above.) Let $\mathrm{d}_{\mathfrak{a}}^{p}: H^{p}(\mathfrak{a}) \rightarrow H^{p+2}(\mathfrak{a})$ be the map induced by the wedge product with

$$
v=\sum_{i=1}^{n} x_{i}^{*} \wedge y_{i}^{*}
$$

Then the diagram

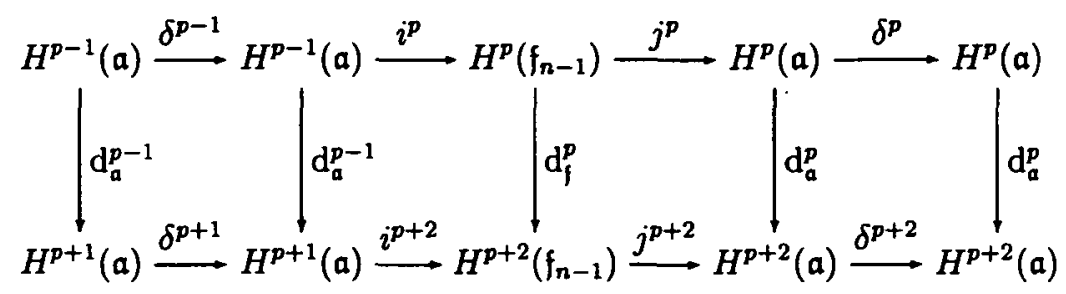

is commutative.

Although the lemma below is well-known (see for instance [8]), we include a proof of this result for completeness.

Lemma 4. Let $a_{n}$ be the 2n-dimensional Abelian Lie algebra generated by $x_{1}, \ldots, x_{n}, y_{1}, \ldots, y_{n}$. Let $v=\sum_{i=1}^{n} x_{i} \wedge y_{i}$. Then, for any $p \geqslant 0$, the map

$$
d_{n}^{p}: \Lambda^{p} a_{n}^{*} \rightarrow \Lambda^{p+2} a_{n}^{*}: f \mapsto f \wedge v
$$

has maximal rank, that is, $\mathrm{d}_{n}^{p}$ is injective for all $0 \leqslant p \leqslant n-1$, and surjective for all $n-1 \leqslant p \leqslant 2 n$.

From these two lemmas, proposition 2 follows at once. For $p \leqslant n-2$, both $\mathrm{d}_{a}^{p-1}$ and $\mathrm{d}_{a}^{p}$ are injective, so the Five Lemma (see [5]) applied to the diagram (4) assures that also $\mathrm{d}_{\mathrm{f}}^{p}$ is injective. For $p \geqslant n-1$, both $\mathrm{d}_{a}^{p-1}$ and $\mathrm{d}_{a}^{p}$ are surjective, and therefore, according to the Five Lemma again, $d_{f}^{p}$ is also surjective.

Proof of LEMma 3. First of all, we show that $\mathrm{d}_{f}^{p+1} i^{p}=i^{p+2} \mathrm{~d}_{\mathfrak{a}}^{p}$. Let $f \in \Lambda^{p} \mathfrak{a}^{*}$. Then

$$
\mathrm{d}_{\mathrm{f}}^{p} i^{p}(f)=i^{p}(f) \wedge z^{*} \wedge z^{* *}+i^{p}(f) \wedge \sum_{i=1}^{n} x_{i} \wedge y_{i}
$$

But $i^{p}(f)\left(x_{i_{1}} \wedge \ldots \wedge x_{i_{s}} \wedge y_{j_{1}} \wedge \ldots \wedge y_{j_{p-s+1}}\right)=0$ for all $s \leqslant p+1,1 \leqslant i_{1}<\cdots<i_{s} \leqslant n$ and $1 \leqslant j_{1}<\cdots<j_{p-s+1} \leqslant n$. Therefore,

$$
i^{p}(f) \wedge z^{*} \wedge z^{* *}=0
$$

Now

$$
\mathrm{d}_{i}^{p} i^{p}(f)=i^{p}(f) \wedge\left(\sum_{i=1}^{n} x_{i}^{*} \wedge y_{i}^{*}\right)=i^{p+2}(f \wedge v)=i^{p+2} \mathrm{~d}_{\mathfrak{a}}^{p} f
$$


Next, we show that $j^{p+2} \mathrm{~d}_{\mathfrak{f}}^{p}=\mathrm{d}_{\mathfrak{a}}^{p} j^{p}$. Let $f$ be a representative of a cohomology class in $H^{p}\left(\mathfrak{f}_{n-1}\right)$. Since $j^{*}$ just means restricting the domain to $\Lambda^{*} \mathfrak{a}$, we have

$$
j^{p+2} \mathrm{~d}_{f}^{p}(f)=j^{p+2}(f \wedge u)=j^{p} f \wedge j^{2} u=-j^{p} \wedge v=-\mathrm{d}_{a}^{p} j^{p} f .
$$

Finally, we show that $\delta^{p+2} \mathrm{~d}_{\mathfrak{a}}^{p}=\mathrm{d}_{\mathfrak{a}}^{p} \delta^{p}$. Let $f \in \Lambda^{p} \mathfrak{a}^{*}$. Since $\delta^{p}$ is nothing but the action induced by the adjoined action of $z$ (see [4]), we have

$$
\delta^{p+2} \mathrm{~d}_{a}^{p}(f)=z \cdot(f \wedge v)=\mathrm{d}_{a}^{p+2} \delta^{p} f+f \wedge(z \cdot v) .
$$

But $z \cdot v=0$, and therefore $\delta^{p+2} \mathrm{~d}_{a}^{p}=\mathrm{d}_{a}^{p+2} \delta^{p}$.

4.1. Proof of lemma 4. We prove lemma 4 by induction on the dimension of $a_{n}$.

For $n=1$, the image of $1 \in \Lambda^{0} \mathfrak{a}_{1}^{*}$ under $\mathrm{d}_{1}^{0}$ is just $x_{1}^{*} \wedge y_{1}^{*}$, so $\mathrm{d}_{1}^{0}$ is injective. Clearly, since $\Lambda^{3} \mathfrak{a}_{1}=\Lambda^{4} \mathfrak{a}_{1}=0, d_{1}^{1}$ and $d_{1}^{2}$ are surjective.

Now suppose this lemma has been proved for even dimensional Abelian Lie algebras up to dimension $2(n-1)$. Fix $p$. When decomposing $\Lambda^{p} \mathfrak{a}_{n}^{*}$ as

$$
x_{1}^{*} \wedge \Lambda^{p-1} a_{n-1}^{*}+y_{1}^{*} \wedge \Lambda^{p-1} a_{n-1}+x_{1}^{*} \wedge y_{1}^{*} \wedge \Lambda^{p-2} a_{n-1}^{*}+\Lambda^{p} a_{n-1}
$$

we see that

$$
\begin{aligned}
\mathrm{d}_{n}^{p}\left(x_{1}^{*} \wedge f\right) & =x_{1}^{*} \wedge \mathrm{d}_{n-1}^{p-1} f & & \text { for } f \in \Lambda^{p-1} \mathrm{a}_{n-1}^{*} \\
\mathrm{~d}_{n}^{p}\left(y_{1}^{*} \wedge f\right) & =y_{1}^{*} \wedge \mathrm{d}_{n-1}^{p-1} f & & \text { for } f \in \Lambda^{p-1} a_{n-1}^{*} \\
\mathrm{~d}_{n}^{p}\left(x_{1}^{*} \wedge y_{1}^{*} \wedge f\right) & =x_{1}^{*} \wedge y_{1}^{*} \wedge \mathrm{d}_{n-1}^{p-2} f & & \text { for } f \in \Lambda^{p-2} \mathfrak{a}_{n-1}^{*} \\
\mathrm{~d}_{n}^{p}(f) & =\mathrm{d}_{n-1}^{p} f+x_{1}^{*} \wedge y_{1}^{*} \wedge f & & \text { for } f \in \Lambda^{p} \mathrm{a}_{n-1}^{*}
\end{aligned}
$$

Therefore, $\mathrm{d}_{n}^{p}$ is injective (or surjective) if and only if both $\mathrm{d}_{n-1}^{p-1}$ and

$$
\alpha^{p}=\left.\mathrm{d}_{n}^{p}\right|_{x_{i}^{*} \wedge y_{i}^{*} \wedge \Lambda^{p-2} a_{n-1}^{*}+\Lambda^{p} a_{n-1}}
$$

are. Note that $\alpha^{p}$ is injective (or surjective) in case both $d_{n-1}^{p}$ and $d_{n-1}^{p-2}$ are.

For $p \leqslant n-2$, the induction hypothesis implies that $d_{n-1}^{p-2}, d_{n-1}^{p-1}$ and $d_{n-1}^{p}$ all are injective, so $\mathrm{d}_{n}^{p}$ is as well. Analogously, for $p \geqslant n$, we know from the induction hypothesis that $d_{n-1}^{p-2}, d_{n-1}^{p-1}$ and $d_{n-1}^{p}$ are surjective, so $d_{n}^{p}$ is too.

For $p=n-1$, the induction hypothesis states that the maps $\mathrm{d}_{n-1}^{n-3}$ and $\mathrm{d}_{n-1}^{n-2}$ are injective, whereas $d_{n-1}^{n-1}$ is surjective. To settle this last case, we need to show that $\alpha^{n-1}$ is injective (or, equivalently, surjective). This is done by showing that

$$
\operatorname{ker} d_{n-1}^{n-3} \cap \operatorname{Im} d_{n-1}^{n-1}=0 .
$$

In fact, we prove slightly more than this in 
LEMMA 5. (With notations as above.) For each $2 \leqslant k \leqslant n$, let

$$
\mathrm{D}_{n}^{k}=\mathrm{d}_{n}^{n+k-2} \ldots \mathrm{d}_{n}^{n-k+2} \text {. }
$$

Then Im $\mathrm{d}_{n}^{n-k} \cap \operatorname{ker} D_{n}^{k}=0$.

Since the proof of lemma 4 for a Lie algebra of dimension $2 n$ only needs lemma 5 for a Lie algebra of dimension $2(n-1)$, we may safely use lemma 4 for Lie algebras of dimension $2 n$ while proving lemma 5 for algebras of dimension $2 n$.

ProOF OF LEMMA 5: We proceed by induction on $n$. For $n=2$ we only need to check the case $k=2$. In this case

$$
\operatorname{Im} \mathrm{d}_{2}^{0}=\left\langle x_{1} \wedge y_{1}+x_{2} \wedge y_{2}\right\rangle
$$

while

$$
\mathrm{d}_{2}^{2}\left(x_{1} \wedge y_{1}+x_{2} \wedge y_{2}\right)=-2 x_{1} \wedge x_{2} \wedge y_{1} \wedge y_{2} \neq 0
$$

Now suppose the lemma has been proven for Abelian Lie algebras up to dimension $2(n-1)$. Let $2 \leqslant k \leqslant n$. We decompose $\Lambda^{n-k}$ and $\Lambda^{n-k+2}$ again as in (5), and use the expressions (6), (7), (8) and (9) for $\mathrm{d}_{n}^{p}$ with respect to these decompositions.

Any element of $\operatorname{Im~}_{n}^{n-k}$ is of the form

$$
x_{1}^{*} \wedge d_{n-1}^{n-k-1} f_{1}+y_{1}^{*} \wedge d_{n-1}^{n-k-1} f_{2}+x_{1}^{*} \wedge y_{1} \wedge\left(f_{4}+d_{n-1}^{n-k-2} f_{3}\right)+d_{n-1}^{n-k} f_{4},
$$

where $f_{1}, f_{2} \in \Lambda^{n-k-1} \mathfrak{a}_{n-1}, f_{3} \in \Lambda^{n-k-2} \mathfrak{a}_{n-1}$ and $f_{4} \in \Lambda^{n-k} \mathfrak{a}_{n-1}$. For the map $D_{n}^{k}$ we find

$$
\mathrm{D}_{n}^{k}\left(x_{1}^{*} \wedge g_{1}\right)=x_{1}^{*} \wedge\left(\mathrm{d}_{n-1}^{n+k-3} \ldots \mathrm{d}_{n-1}^{n-k+1} g_{1}\right)=x_{1}^{*} \wedge \mathrm{D}_{n-1}^{k} g_{1}
$$

for any $g_{1} \in \Lambda^{n-k+1} a_{n-1}$, and analogously

$$
\mathrm{D}_{n}^{k}\left(y_{1}^{*} \wedge g_{2}\right)=y_{1}^{*} \wedge\left(\mathrm{d}_{n-1}^{n+k-3} \ldots \mathrm{d}_{n-1}^{n-k+1} g_{2}\right)=y_{1}^{*} \wedge \mathrm{D}_{n-1}^{k} g_{2}
$$

for any $g_{2} \in \Lambda^{n-k+1} \mathfrak{a}_{n-1}$. For $D_{n}^{k}$ applied to the last two parts in the decomposition of $\Lambda^{n-k+2} a_{n}$ one proves easily that

$$
\mathrm{D}_{n}^{k}\left(x_{1}^{*} \wedge y_{1}^{*} \wedge g_{3}+g_{4}\right)=x_{1}^{*} \wedge y_{1}^{*} \wedge\left((k-1) \mathrm{D}_{n-1}^{k-1} g_{4}+\mathrm{D}_{n-1}^{k-1} \mathrm{~d}_{n-1}^{n-k} g_{3}\right)+\mathrm{d}_{n-1}^{n+k-2} D_{n-1}^{k-1} g_{4}
$$

for any $g_{3} \in \Lambda^{n-k} a_{n-1}$ and any $g_{4} \in \Lambda^{n-k+2} a_{n-1}$.

Now suppose $g=x_{1}^{*} \wedge g_{1}+y_{1}^{*} \wedge g_{2}+x_{1}^{*} \wedge y_{1}^{*} \wedge g_{3}+g_{4}$ is contained in Im $\mathrm{d}_{n}^{n-k} \cap$ ker $\mathrm{D}_{n}^{k}$. Then we have

$$
\begin{aligned}
\mathrm{D}_{n-1}^{k} g_{1} & =0 \\
\mathrm{D}_{n-1}^{k} g_{2} & =0 \\
(k-1) \mathrm{D}_{n-1}^{k-1} g_{4}+\mathrm{D}_{n-1}^{k-1} \mathrm{~d}_{n-1}^{n-k} g_{4} & =0 \\
\mathrm{~d}_{n-1}^{n+k-2} D_{n-1}^{k-1} g_{4} & =0
\end{aligned}
$$


and there exists a $f=x_{1}^{*} \wedge f_{1}+y_{1}^{*} \wedge f_{2}+x_{1}^{*} \wedge y_{1}^{*} \wedge f_{3}+f_{4} \in \Lambda^{n / 2-2} a_{n}$ such that

$$
\begin{aligned}
d_{n-1}^{n-k-1} f_{1} & =g_{1}, \\
d_{n-1}^{n-k-1} f_{2} & =g_{2}, \\
f_{4}+d_{n-1}^{n-k-2} f_{3} & =g_{3}, \\
d_{n-1}^{n-k} f_{4} & =g_{4} .
\end{aligned}
$$

The induction hypothesis implies that the systems of equations (10), (14) and (11), (15) do not have a solution except the trivial one. From equations (12) and (17) it follows that

$$
\mathrm{D}_{n-1}^{k-1} \mathrm{~d}_{n-1}^{n-k}\left((k-1) f_{4}+g_{3}\right)=0 .
$$

Stated otherwise, $\mathrm{d}_{n-1}^{n-k}\left((k-1) f_{4}+g_{3}\right) \in \operatorname{ker} D_{n-1}^{k-1}$. The induction hypothesis now states that $\mathrm{d}_{n-1}^{n-k}\left((k-1) f_{4}+g_{3}\right)=0$, and thus $g_{3}=-(k-1) f_{4}$ by the injectivity of $\mathrm{d}_{n-1}^{n-k}$. Substituting $g_{3}$ in (16) yields

$$
d_{n-1}^{n-k-2} f_{3}=k f_{4}
$$

while (17) combined with (13) gives

$$
\mathrm{d}_{n-1}^{n+k-2} D_{n-1}^{k-1} d_{n-1}^{n-k} f_{4}=D_{n-1}^{k+1} f_{4}=0
$$

The induction hypothesis again yields $f_{4}=0$, and therefore $g_{3}=g_{4}=0$.

\section{EXAMPLES}

5.1. A family OF THREe-STEP NILPOTENT LIE ALgebras. Let $n \geqslant 1$ and $\mathfrak{b}_{n}$ the Lie algebra of dimension $2 n+2$ with a basis

$$
\left\{x_{1}, \ldots, x_{n}, y_{1}, \ldots y_{n}, w, z\right\}
$$

and Lie bracket

$$
\left[z, x_{i}\right]=y_{i}, \quad\left[x_{i}, y_{i}\right]=w
$$

for all $1 \leqslant i \leqslant n$. Then $\mathfrak{b}_{n}$ is an extension of $\langle z\rangle$ by the Heisenberg Lie algebra $\mathfrak{h}_{n}$. The Betti numbers of the quotient algebra $\mathfrak{f}_{n}=\mathfrak{b}_{n} /\langle w\rangle$ are given by

$$
\beta_{p}\left(\mathfrak{f}_{n}\right)=\left(\begin{array}{c}
n+1 \\
\left\lfloor\frac{1+p}{2}\right\rfloor
\end{array}\right)\left(\begin{array}{c}
n \\
\left\lfloor\frac{p}{2}\right\rfloor
\end{array}\right),
$$

where $\lfloor x\rfloor$ denotes the integer part of $x$ (see [2]). The main theorem allows us to compute the Betti numbers of $\boldsymbol{b}_{n}$ in terms of the cohomology of $\mathfrak{f}_{n}$. 26.

The table below lists the Betti numbers (or, at least, half of them) up to dimension 
As illustrated in the figure below (showing the distribution of the Betti numbers of $\beta_{n}$ for $n=3,4,5,6,10$ and 40 ), these Betti numbers do not have a unimodal distribution. It is easy to prove that

$\beta_{n}\left(\mathfrak{b}_{n}\right)-\beta_{n+1}\left(\mathfrak{b}_{n}\right)= \begin{cases}\frac{n !(n+1) !}{(n / 2) !(n / 2+1) !(n / 2+1) !(n / 2+2) !}\left(\frac{n}{2}-2\right) & \text { for } n \text { even, } \\ \frac{n !(n+1) !}{((n-1) / 2) !((n+1) / 2) !((n+3) / 2) !((n+3) / 2) !} \frac{n-1}{2} & \text { for } n \text { odd. }\end{cases}$

The sign of this difference is determined by a linear polynomial; for $n=1$ or $n=4$ we see that $\beta_{n}\left(\mathfrak{b}_{n}\right)=\beta_{n+1}\left(\mathfrak{b}_{n}\right)$, for $n=2$ we have $\beta_{n}\left(\mathfrak{b}_{n}\right)<\beta_{n+1}\left(\mathfrak{b}_{n}\right)$ and for $n=3$ or $n \geqslant 5$ we have $\beta_{n}\left(\mathfrak{b}_{n}\right)>\beta_{n+1}\left(b_{n}\right)$, thus implying non-unimodality. An analysis along the same lines allows us to determine exactly the number $p$ such that $\beta_{p-1}\left(b_{n}\right)<\beta_{p}\left(b_{n}\right)$ but $\beta_{p}\left(b_{n}\right)>\beta_{p+1}\left(b_{n}\right)$.

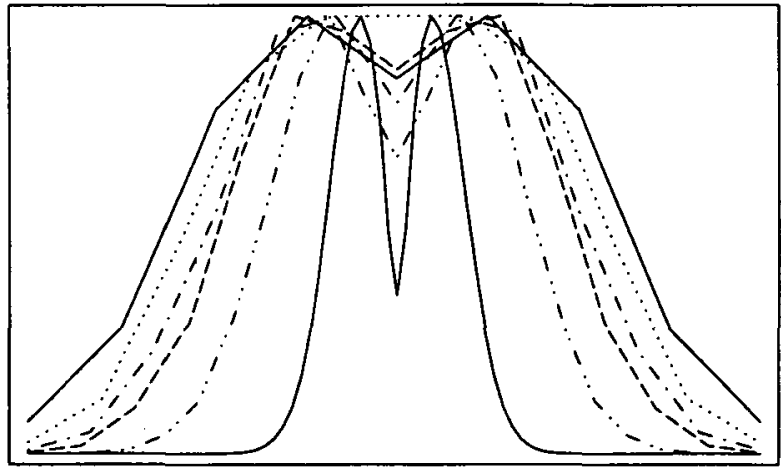

5.2. COHOMOLOGY OF THE TWISTED STANDARD FILIFORM LIE ALgEBRAS. Let $\mathfrak{g}_{n}$ be the twisted standard filiform Lie algebra of dimension $n+2$ given in (2). Then $g_{n}$ is an extension of $\langle z\rangle$ by the Heisenberg algebra $\mathfrak{h}_{n}$, and $\mathfrak{g}_{n} /\langle w\rangle$ is just the standard filiform Lie algebra $f_{n}$ of dimension $n+1$. According to [3], the Betti numbers of this Lie algebra are given by the formula

$$
\beta_{p}\left(f_{n}\right)=\kappa_{p}+\kappa_{p-1}
$$

where

$$
\kappa_{p}=\#\left\{\left(\alpha_{1}, \ldots, \alpha_{p}\right) \in \mathbb{Z}^{p} \mid 1 \leqslant \alpha_{1}<\cdots<\alpha_{p} \leqslant n \text { and } \sum_{i} \alpha_{i}=\left\lfloor\frac{i(n+1)}{2}\right\rfloor\right\} .
$$

Again, the main theorem tells us how to compute the Betti numbers of $\mathfrak{g}_{\boldsymbol{n}}$ in terms of the Betti numbers of $\mathfrak{f}_{n}$. For the Betti numbers (at least, half of them) of the algebras $g_{n}$ up to dimension 26 we find 


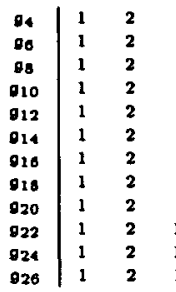

Again, we see that the Betti numbers of these low-dimensional examples follow a non-unimodal distribution. The figure below shows the distribution of the Betti numbers of $\mathfrak{g}_{n}$ for $n=3,4,7,10$ and 12. However, a proof of this fact would probably involve partition functions and, in particular, the way their coefficients grow with respect to one another, which is far beyond the scope of this text.

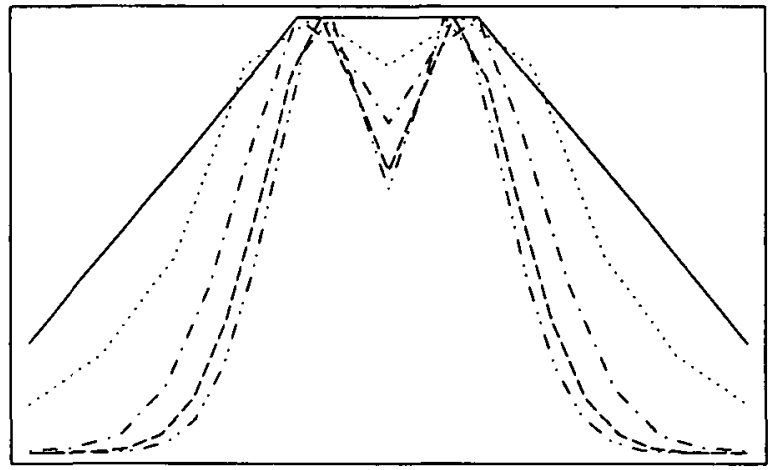

\section{REFERENCES}

[1] G.F. Armstrong, 'Unimodal Betti numbers for a class of nilpotent Lie algebras', Comm. Algebra 25 (1997), 1893-1915.

[2] G.F. Armstrong, G. Cairns and B. Jessup, 'Explicit Betti numbers for a family of nilpotent Lie algebras', Proc. Amer. Math. Soc. 125 (1997), 381-385.

[3] G.F. Armstrong and S. Sigg, 'On the cohomology of a class of nilpotent Lie algebras', Bull. Austral. Math. Soc. 54 (1996), 517-527.

[4] J. Dixmier, 'Cohomologie des algèbres de Lie nilpotentes', Acta Sci. Math. 16 (1955), 246-250.

[5] S. Mac Lane, Homology, Die Grundlehren der Math. Wissenschaften 114 (Springer-Verlag, Berlin, Heidelberg, New York, 1975).

[6] L.J. Santharoubane, 'Cohomology of Heisenberg Lie algebras', Proc. Amer. Math. Soc. 87 (1983), 23-28.

[7] P. Tirao, 'A refinement of the toral rank conjecture for 2-step nilpotent Lie algebras', Proc. Amer. Math. Soc. 128 (2000), 2875-2878.

[8] R.O. Wells, Differential analysis on complex manifolds, Graduate texts in mathematics 65 (Springer-Verlag, Berlin, Heidelberg, New York, 1980). 
Katholieke Universiteit Leuven (KULAK)

Etienne Sabbelaan 53

8500 Kortrijk

Belgium

e-mail: hannes.pouseele@kulak.ac.be 